\title{
Va \\ Les documents polycopiés, un instrument au service des pratiques enseignantes pour ajuster les compétences scripturales des élèves et les contenus enseignés
}

\author{
Mehdi Khaneboubi, laboratoire STEF (IFÉ - ENS de Lyon) \\ Camille Roux-Goupille, Univ Paris Est Creteil, LDAR
}

Linterprétation des discours de 46 enseignant.e.s de lycées généraux, technologiques et professionnels nous fait considérer la production de documents photocopiés comme une adaptation des contenus enseignés aux capacités de lecture et d'écritures des élèves. Les compétences scripturales des élèves orientent la stratégie d'enseignement des enseignant.e.s et la nature des photocopies. Selon quills considèrent que leurs élèves ont besoin de s'exercer à lire ou à écrire, ils conçoivent un document adapté à cette estimation. Lorsque les élèves sont de bons lecteurs, le support devient invisible et la stratégie d'enseignement et de conception de la photocopie est focalisée sur les contenus uniquement.

\section{Introduction}

Considérer des technologies plus anciennes et mieux instituées pour porter un regard différent sur les plus récentes est particulièrement fécond. Si on s'intéresse aux technologies en usage, on remarque que l'impact social des technologies les plus récentes est souvent moins important que celui des plus anciennes (Edgerton $\&$ Pestre, 1998). Cette approche, qui considère les usages plutôt que les innovations, permet de porter une plus grande attention sur les technologies ordinaires et omniprésentes plutôt que sur les technologies à la mode. Edgerton (Edgerton \& Jeanmougin, 2013) plaide donc pour la reconnaissance de l'importance des techniques dites anciennes. Il incite à considérer avec précaution les discours sur «l'innovation» qui se fondent moins sur l'efficacité de fait des techniques nouvelles que sur des considérations d'ordre idéologique. Mais cette analyse montre avant tout que les technologies anciennes et nouvelles cohabitent, interagissent, voire s'hybrident, ce qui rejoint les travaux de Goody concernant les technologies de l'écrit (Mayor \& Chevalier, 2008).

En 1986, Cuban a décrit comment aux États-Unis les enseignant-e.s utilisaient les technologies majeures du $20^{\mathrm{e}}$ siècle. Les technologies considérées (cinéma, 
radio, télévision, ordinateur) ont été peu utilisées par les enseignant·e.s en raison, d'après lui, d'un manque de compétences techniques, de coûts d'entretien trop élevés pour les institutions, de disponibilités relatives, et d'une inadéquation avec les curriculums (Cuban, 1986). Les enseignant.e.s hybrident leurs pratiques avec les technologies lorsqu'elles les aident à résoudre des problèmes qui leur paraissent importants et évitent d'éroder leur autorité dans la classe. Ils et elles laissent donc de côté les technologies qui augmentent leur charge de travail sans bénéfice pour leurs élèves ou qui affaiblissent leur contrôle de la classe. De ce point de vue, on comprend que les enseignant.e.s aient le plus souvent recours à des technologies plus anciennes, mieux éprouvées et plus fiables (Cuban, 2001).

Cèbe et Goigoux (2007) rejoignent cette analyse en considérant l'enseignement comme une activité médiatisée par des dispositifs techniques. Selon eux, les enseignant.e.s ne les jugent pas uniquement sur la base de leurs effets supposés sur les apprentissages. Ils et elles les adoptent uniquement si la technologie est compatible avec leurs conceptions et leurs pratiques habituelles. Les enseignant.e.s les rejettent si elles suscitent un sentiment d'insécurité ou accroissent la pénibilité de leur travail. Dans cette perspective, les outils doivent ainsi être en accord avec les conceptions pédagogiques et les compétences professionnelles des enseignant.e.s sans négliger les caractéristiques des élèves ni les connaissances à enseigner.

En cherchant à comprendre les usages peu importants d'ordinateurs dans un contexte pourtant saturé en technologies informatisées, on a décrit (Khaneboubi, 2009) comment les actions techniques et pédagogiques des enseignant.e.s dans la classe étaient les deux faces d'une même pratique. Ainsi on constatait statistiquement que les enseignant.e.s utilisant le plus souvent les ordinateurs en classe étaient significativement plus expérimenté.e.s - et donc au bénéfice d'un savoir-faire pédagogique et didactique conséquent - que les enseignant.e.s les plus fraichement recruté.e.s qui avaient une plus grande familiarité avec l'informatique, mais employaient rarement les ordinateurs avec leurs élèves. Les enseignant.e.s observé.e.s, utilisatrices et utilisateurs d'ordinateurs, étaient suffisamment avancé.e.s pédagogiquement pour intégrer les contraintes techniques dans le déroulement de la classe. Les autres enseignant.e.s utilisant peu les ordinateurs déclaraient que les problèmes techniques sabordaient le cours. On en concluait que les compétences pédagogiques des enseignant.e.s n'étaient pas à même de se mettre en ouvre sans connaissances techniques et inversement: en pratique, dans la classe, ces deux types de savoir-faire étaient indissociables.

Le prolongement de cette idée permet de soutenir qu'il n'y a pas, dans la perception des enseignant.e.s, de distinction entre le support de la connaissance et la connaissance dès lors que les technologies sont maitrisées. Autrement dit, la matérialité des documents employés en classe constitue pratiquement les savoirs. Lorsque les technologies sont incorporées, les manipulations techniques sont vécues comme des manipulations de contenus, notions, etc. Si les technologies employées ne font pas obstacle, le médium n'est plus perçu, seuls les savoirs 
sont considérés. Cette perspective fait écho, dans un contexte plus global, aux thèses de Goody (1979): l'interaction entre les informations et les supports qui les véhiculent, développe de nouvelles possibilités de traitement qui génèrent des évolutions dans la cognition.

Un travail sur les technologies plus anciennes permet d'élaborer cette question et de saisir les nécessités pratiques des enseignant.e.s et les réponses qu'ils y apportent avec d'autres technologies que celles informatisées. C'est l'objet de ce texte qui porte sur les usages scolaires des documents polycopiés. Cette étude s'inscrit dans le projet ReVEA traitant du travail des enseignant.e.s avec les ressources: conceptions, recherches, sélections, modifications et recompositions (Bruillard, 2019). Une partie du projet avait pour objectif de dresser un panorama des ressources potentiellement utilisables par les enseignant.e.s et d'examiner les usages effectifs. Les photocopies, comme objets à la jonction entre les contenus et les élèves, sont au centre des pratiques enseignantes et constituent un objet de recherche peu étudié. Dans quel écosystème documentaire naissent les photocopies? Pourquoi est-il impossible pour les enseignant.e.s de se passer des photocopies? Dans quelles stratégies d'enseignement s'inscrit l'usage des photocopies?

\section{Natures et rôles des photocopies}

Aujourd'hui, dans les écoles, collèges et lycées de France, les techniques de reprographie sont centrales. Les documents photocopiés sont mobilisés par tous les enseignant.e.s dans toutes les disciplines et tous les niveaux. Parmi l'ensemble de documents consultés, manipulés et produits par les enseignant.e.s, les photocopies constituent des objets particuliers. D'abord parce qu'il s'agit de documents le plus souvent composés par les enseignant.e.s, semblables à un patchwork, avec une cohérence propre, issue de multiples sources qui en font des originaux. Ensuite, parce qu'il s'agit d'écrits remis aux élèves et produits dans cette optique. Pourtant, on trouve peu d'études sur les photocopies.

Dans l'enseignement du français à l'école primaire en Suisse Romande, Thévenaz-Christen (2014) indique que les photocopies ont des structures sophistiquées combinant des écrits et des illustrations d'origines diverses et souligne leur caractère multifonctionnel:

- duplication de texte ou d'extraits,

- aide-mémoire,

- exercice ou document issu d'internet,

- document intégral issu d'une maison d'édition,

- écriture directement sur le support.

Dans le cadre de l'enseignement du français, Plane et Schneuwly (2000) ont estimé que les outils de l'enseignant.e étaient peu considérés dans les approches didactiques disciplinaires et dans les approches pédagogiques. Ils ont esquissé une 
définition des outils d'enseignement du français comme «tout artefact introduit dans la classe de français servant l'enseignement/apprentissage des notions et capacités» (p. 5). Ils jugent pertinente l'exploration des différentes conceptions de ces outils. De façon similaire, Nonnon (2000) examine le cas du tableau noir et affirme qu'il fait partie des «outils méconnus, invisibles et illégitimes de la pratique» (p. 87) alors qu'il s'agit d'un instrument à la jonction entre la planification et l'improvisation enseignante illustrant le «bricolage interprétatif et stratégique» (p. 85) de leur pratique. Cette conception fait écho aux documents polycopiés académiquement déconsidérés bien que faisant partie intégrante de l'environnement scolaire comme médium entre les contenus et les élèves.

Dans le contexte de formation d'enseignant.e.s belges en sciences de la vie, Poffé, Laschet et Hindrycks (2015) montrent que les supports photocopiés fournis aux élèves sont conçus avant tout pour aider les enseignant.e.s en classe à délivrer leurs leçons plutôt que pour structurer et faciliter les apprentissages des élèves. Ainsi, le style rédactionnel, les illustrations présentées (dessins, graphiques, schémas), les paratextes associés et l'absence fréquente de synthèse, contribuent à rendre difficile un travail en autonomie, indépendamment de l'usage qui en est fait en classe. Ces conclusions rejoignent en partie le travail de Balcou-Debussche (2007) dans l'enseignement primaire en France qui constate que les photocopies sont un organisateur des pratiques professionnelles de l'enseignant. Ce phénomène est similaire à celui que Choppin (2005) remarque concernant l'évolution formelle des manuels scolaires dont l'évolution vers une grande modularité des manuels prévoit des usages partiels, des recompositions par les enseignant.e.s.

Dès 1974 avant la mise en place de la réforme Haby dite du "collège unique", la reproduction de documents papier est banalisée. 250 millions de copies par an sont faites dans les établissements du second degré. Alors que $43 \%$ d'établissements utilisaient encore des duplicateurs à alcool, $24 \%$ ont déjà des photocopieurs. À l'époque, la moitié des documents reproduits sont des documents originaux (sujets et exercices élaborés par les professeur.e-s), $31 \%$ des documents officiels et $6 \%$ des manuels scolaires. Parmi les reproductions d'œuvres protégées par le droit d'auteur, la proportion de manuels scolaires varie selon le type d'établissement: $61 \%$ dans les lycées classiques et modernes et seulement $38 \%$ dans les lycées techniques et polyvalents (Thouvenin, 1975).

Une étude australienne (Horsley, 2012; Horsley \& Walker, 2011) sur les photocopies a cherché à qualifier la viabilité de la substitution des documents papier par des documents numériques en contexte scolaire. En 2011, pour la première fois, l'Australie a mis en place des programmes scolaires communs à toutes les écoles du pays. Pour fournir des ressources pédagogiques aux élèves et aux enseignant.e.s dans la mise en œuvre des nouveaux programmes, une société a développé un répertoire national de ressources éducatives en ligne. De plus, chaque élève australien âgé de 9 à 12 ans a été doté d'un ordinateur portable. Horsley (2012) étudie les documents photocopiés par les professeur.e.s dans les 
enquêtes réalisées par la société de gestion des droits d'auteurs. Il estime que seulement $3 \%$ des photocopies proviennent des ressources en ligne du répertoire. Les manuels scolaires et documents papier constituent la source quasi unique des photocopies et donc des ressources d'enseignement et d'apprentissage en classe. Il s'interroge donc sur la viabilité d'un unique catalogue centralisé comme source de documents pour les enseignant.e.s.

Un des apports de ce travail est de considérer les enquêtes réalisées par la société de gestion de droit d'auteur comme une source de données quantitatives reflétant les activités des enseignant.e.s. En France, la société responsable de la gestion du droit de reproduction, nommé Centre français d'exploitation du droit de copie (CFC), perçoit et redistribue des redevances collectées auprès d'établissements scolaires et universitaires, d'entreprises et d'administrations. $70 \%$ des sommes perçues au titre de la reprographie par le CFC proviennent des établissements scolaires. Pour redistribuer ces fonds, le CFC réalise des enquêtes annuelles par sondage en collèges et lycées. Dans le cadre d'une collaboration avec le projet ReVEA, une base de données produite par les enquêtes des années 2011-2012 et 2012-2013 a été étudiée (Boelaert \& Khaneboubi, 2016). Une analyse de données a révélé des inégalités extrêmes. Ainsi, les huit éditeurs les plus photocopiés totalisent $70 \%$ des actes de photocopie en 2013 alors que la même année $53 \%$ des éditeurs ne sont concernés que par un seul acte de photocopie. Par ailleurs, l'essentiel des photocopies concerne des ouvrages scolaires. Le français, l'histoire-géographie et les langues déclarent plus d'actes de photocopies sous droits d'auteurs que les disciplines scientifiques. Du point de vue des pratiques enseignantes ces données montrent que les manuels sont la première source des photocopies.

\section{Méthodologie et déroulement}

Entre 2015 et 2016, un travail qualitatif a été conduit dans quatre lycées polyvalents d'Ile-de-France (accueil des élèves dans des sections générales, techniques et professionnelles désignées ci-dessous Lycées A, B, C et D). Il s'agissait principalement de rencontrer des enseignant.e.s dans les services de reprographie et en salle des professeur.e.s. Une trame d'entretien portant sur les usages des photocopies a été produite pour les enseignant.e.s, les gestionnaires et les responsables des services de reprographie (cf. annexe). Il fallait en outre collecter des éléments contextuels sur les établissements comme le nombre d'élèves et d'enseignant.e.s, leur localisation, les filières particulières, le devenir des élèves. Dans une perspective d'ethnographie scolaire, des observations en salle de reprographie ont permis de conduire des entretiens contextualisés de novembre 2014 à mars 2015, deux fois par mois dans les lycées $\mathrm{A}$ et $\mathrm{B}$, une demi-journée dans le lycée $\mathrm{D}$ et deux demijournées dans le lycée $C$ en 2016. Au final on dispose de 46 entretiens avec des enseignant.e.s, 4 entretiens avec des gestionnaires et 4 avec des responsables de 
services de reprographie. Les entretiens ont été enregistrés et retranscrits pour être analysés.

L'analyse des entretiens s'est faite en s'inspirant de la théorie ancrée (grounded theory, Glaser \& Strauss, 2010) c'est-à-dire en relevant des éléments de sens pour construire les thèmes généraux présentés par la suite. L'interprétation de ces données repose sur une analyse de notre objet «comme relevant non d'une science expérimentale en quête de loi, mais d'une science interprétative en quête de sens", issue de la perspective théorique de Geertz (1998). Il s'agissait donc pour nous, d'ordonner ce qui a été dit en:

[essayant] de lire (au sens de "construire une lecture de») un manuscrit étranger, défraîchi, plein d'ellipses, d'incohérences, de corrections suspectes et de commentaires tendancieux, et écrit non à partir de conventions graphiques normalisées, mais plutôt de modèles éphémères de formes de comportement (p. 80).

Le tableau 1 (ci-dessous) présente les caractéristiques générales de ces établissements. On remarquera les quantités importantes de copies réalisées. Tous les établissements sont situés en zone urbaine; si aucun n'est classé en zone d'éducation prioritaire (ZEP), en revanche trois lycées sont dits polyvalents, et un seul prépare les élèves uniquement à des baccalauréats généraux et techniques. Les quatre lycées délivrent des diplômes de techniciens du supérieur (brevet de technicien du supérieur - BTS) et deux d'entre eux disposent de Classes préparatoires aux grandes écoles (CPGE). De par leur nombre d'enseignant.e.s et d'élèves, ces établissements sont comparables, ce qui permet d'assurer une relative homogénéité des établissements de l'enquête.

Tableau 1: Profils des établissements ayant participé à l'enquête. Les valeurs sont approximatives et basées sur des déclarations sauf pour le lycée $C$ où elles proviennent de documents de gestion.

\begin{tabular}{|c|c|c|c|c|}
\hline & Lycée A & Lycée B & Lycée C & Lycée D \\
\hline $\begin{array}{c}\text { Nombre } \\
\text { d'enseignant·e•s }\end{array}$ & 130 & 110 & 140 & 150 \\
\hline Nombre d'élèves & 1200 & 1000 & 1100 & 1700 \\
\hline Nombre de copies & $\begin{array}{c}100000 \text { lors d'une semaine } \\
\text { chargée }\end{array}$ & $\begin{array}{l}8 \text { million par } \\
\text { an }\end{array}$ & $\begin{array}{c}\text { entre } 50000 \text { et } 70000 \text { par } \\
\text { semaine }\end{array}$ & $\cdot$ \\
\hline Budgets alloués & $\begin{array}{l}\text { Un quart du budget } \\
\text { d'enseignements }\end{array}$ & $\cdot$ & 80000 euros par an & $\begin{array}{c}40000 \text { euros par an } \\
\text { hors papier }\end{array}$ \\
\hline
\end{tabular}

Les photocopies constituent un sujet conflictuel dans les établissements. Si les enseignant.e.s parlent volontiers des photocopies, ils et elles sont souvent réticent.e.s à donner un exemplaire de leurs documents à l'enquêteur comme prévu initialement. À l'échelle de l'établissement le sujet est hautement sensible et potentiellement source de conflits. La question des délais et de l'organisation 
de procédures en est un exemple. Les reprographies sont des lieux importants dans la vie des établissements que nous avons rencontrés: lieu de vie où l'on vient bavarder et boire le café (Lycée B), en accès direct avec la salle des professeur.e.s (Lycée C), passage obligé tous les matins (lycée C).

Les gestionnaires que nous avons rencontré.e.s ont manifesté beaucoup d'intérêt pour la question et toutes et tous cherchent des solutions d'économie comme l'instauration de quotas, la limitation des copies couleur, l'incitation aux copies recto-verso systématiques ou des négociations serrées lors de l'établissement des contrats de location des machines. Les enseignant.e.s sont d'ailleurs mobilisé.e.s sur cette question, ils et elles ont souvent signifié ne pas faire une consommation de photocopies déraisonnable, par exemple en dupliquant en noir et blanc alors que de la couleur aurait été préférable, des recto-verso ou des formats réduits. Les gestionnaires estiment que les travaux de reprographie sont en augmentation constante. Ils et elles ont aussi indiqué que les enseignant.e.s des formations relevant de l'enseignement supérieur sont celles et ceux qui photocopient le plus, et les enseignant.e.s des disciplines littéraires davantage que les scientifiques.

\section{Résultats}

Un échantillonnage de l'écrit

Les documents remis aux élèves sont conçus avec un traitement de texte et imprimés pour les élèves par le service de reprographie d'un établissement ou avec un photocopieur en libre-service. Les documents produits par les enseignant.e.s appartiennent à plusieurs catégories que l'on peut classer en cinq principales:

- les cours,

- les fiches de travaux pratiques ou d'activité,

- les documents: texte, image, plan, documentation technique...

- les exercices,

- les évaluations.

Les documents reprographiés remis aux élèves sont composés en premier lieu par des extraits de manuels issus d'autres manuels que ceux choisis par le lycée pour les élèves: des spécimens, des éditions plus anciennes ou étrangères en langue par exemple. Les spécimens que reçoivent les enseignant.e.s constituent la première source de documentation des enseignant.e.s après le manuel choisi dans leur lycée. Cet envoi d'ouvrages aux enseignant.e.s se fait en échange de réponse à des enquêtes quantitatives annuelles mise en œuvre par les éditeurs scolaires. La plus connue est le questionnaire du GIDEC (groupement d'information d'éditeurs classiques).

La deuxième source de documents constituant les polycopiés pédagogiques reste le moteur de recherche Google. À l'exception de Google recherche d'images, ne sont jamais mentionnés ni les fonctions de recherche avancée, ni d'autres 
moteurs de recherches. Les enseignant.e.s déclarent simplement chercher «dans Google». Les sites consultés sont très rarement cités. En revanche, ils et elles déclarent choisir des documents «intéressants», "pertinents» et indiquent ainsi implicitement réaliser une évaluation des documents qu'ils et elles choisissent.

Deux éléments principaux sont présentés par les enseignant.e.s pour expliquer l'importance qu'ont les photocopies dans leur travail. D'abord, l'absence ou les défaillances des manuels scolaires sont les facteurs le plus souvent mis en avant. Ensuite, c'est le rapport à l'écrit des élèves, c'est-à-dire leurs capacités scripturales, qui oriente la conception d'un polycopié: les photocopies permettent d'adapter ce qui est enseigné au niveau de lecture et d'écriture des élèves. Nous allons voir comment, dans les discours d'enseignant.e.s de lycée que nous avons rencontré.e.s, ces deux éléments expliquent l'importance qu'ont les photocopies.

\section{Un substitut au manuel}

Une enseignante d'anglais dans l'enseignement professionnel expliquait comment, pour ses cours d'anglais en BTS industriel qui relève de l'enseignement supérieur, elle s'accommodait de l'absence de manuel: «[comme il n'existe pas de manuel] alors, je trouve sur internet, voilà je vais piocher un peu partout». Il peut aussi s'agir de pallier une absence de manuels due au fait que les élèves n'amènent pas leur exemplaire: "Quand ils n'ont pas leur matériel, c'est embêtant, ils travaillent avec le voisin, ils bavardent». Il est aussi possible que les élèves soient dispensé.e.s d'amener le manuel, ainsi un enseignant de Physique-Chimie photocopie le manuel du lycée parce que les élèves ne l'ont pas, «trop lourd». Il prévoit donc toujours des photocopies du manuel du lycée, car il estime que comme «la république le paye» il faut l'utiliser même s'il ne correspond pas toujours à ce qu'il avait envie de faire.

Lorsque le manuel existe, mais n'est pas employé, c'est souvent lié aux programmes scolaires. Ainsi, en lettres, les manuels ne sont pas une source privilégiée, comme le présentait une enseignante:

[...] [E]n français on utilise beaucoup plus les photocopies parce qu'on ne suit pas le manuel comme en histoire ou en maths. [...] Nous on travaille par objet d'étude: le théâtre, l'argumentation, la poésie [...]. En lettres dans la mesure où il y a une infinité de textes et où on nous laisse une grande liberté pour travailler selon nos goûts, on fait beaucoup plus de photocopies.

Les enseignant.e.s font référence implicitement à la qualité des manuels au regard de leur liberté et leur préférence. Ainsi, un professeur de sciences économique et sociale (SES) de lycée expliquait: «dans la mesure où les documents des manuels ne sont pas tout le temps satisfaisants, je vais récupérer dans d'autres manuels, j'ai besoin de photocopies». Un enseignant de sciences et technique industriel (STI) présentait cette question en prenant le cas de l'enseignement de l'énergie; 
il estimait que le livre avait une présentation sous forme de liste comme dans un catalogue. Il considérait plus facile de s'approprier les contenus dans un contexte précis:

Par exemple en présentant l'île de Hierro [aux Canaries] qui utilise différentes sources d'énergie alors que dans le livre ils vont pas faire forcément... ce ne sera pas appliqué à ma pédagogie. Par contre, le livre peut servir pour les exercices, mais pas pour suivre un cours. Le manuel n'a pas forcément notre progression.

Une enseignante de sciences physiques affirmait qu'elle s'inspirait de plusieurs manuels scolaires:

[...] pour faire quelque chose qui nous correspond aussi, parce qu'on peut très bien lire un cours sur un manuel et avoir une vision qui n'est pas forcément celle du manuel et une façon d'envisager le cours qui n'était pas celle-là et avec laquelle on ne sera pas à l'aise si on fait celle du manuel scolaire.

En somme, l'utilisation de photocopies est expliquée en faisant référence à l'absence ou à l'inadaptation du manuel au contexte d'enseignement, mais aussi à des goûts, des envies et des préférences portant sur des contenus à enseigner et relevant de l'expertise. Souvent les enseignant.e.s font référence à une dimension personnelle exprimée comme un trait collectif: «faire quelque chose qui nous correspond», «notre progression», «travailler selon nos goûts».

\section{Des stratégies d'enseignement adaptées au rapport à l'écrit des élèves}

Le critère qui explique le mieux l'usage et la nature des documents photocopiés réside dans le rapport à la lecture et à l'écriture des élèves. Ce que disent les enseignant.e.s que nous avons rencontré.e.s c'est que les photocopies sont un moyen d'ajustement, une interface entre les contenus et les capacités de lecture et d'écriture des élèves. Ainsi les documents polycopiés sont considérés comme particulièrement indispensables si les élèves ne peuvent pas lire et écrire beaucoup. Un enseignant de mathématiques et de sciences en lycée professionnel estime que ses élèves ont trop de difficultés à lire et à écrire pour pouvoir faire cours autrement:

[En sciences], c'est quasiment impossible de faire cours sans les photocopies avec ces élèves. Les photocopies sont fondamentales. Ce serait différent s'il s'agissait d'élèves que l'on peut faire écrire, mais on ne peut pas dicter ils sont trop lents. Déjà leur faire lire un texte c'est compliqué, ils se fatiguent vite.

Dans une logique similaire, mais centrée sur l'écriture plus que sur la lecture, un enseignant en sciences et techniques de l'industrie et du développement durable (STI2D) met en avant les difficultés d'écriture de ses élèves, et pourquoi les 
photocopies qu'il leur remet sont peu nombreuses et visent à susciter un travail d'écriture:

En [Sciences et Techniques Industrielles - STI] on a des élèves qui ont du mal [...]. Ils viennent en STI parce qu'ils n'ont pas pu aller en [section scientifique générale]. C'est des gamins qui ont du mal à synthétiser. Leur donner un gros paquet de photocopies [ça ne sert à rien]. Il faut les faire écrire qu'ils puissent synthétiser, après c'est cognitif. Qu'il y ait un geste: j'entends quelque chose et je l'écris sur un support.

Ensuite on rencontre des enseignant.e.s, souvent dans les sections de l'enseignement supérieur (BTS ou CPGE), qui ne mentionnent pas la question du rapport à l'écrit et ne prennent en considération que les contenus. Par exemple cette enseignante de Français en BTS déclarait en parlant des documents qu'elle préparait pour ses élèves: "J'ai pris [ces textes] dans un manuel que j'ai reçu. Ils ont un intérêt par rapport à la problématique et sont susceptibles de leur plaire». Un des textes porte sur un homme qui raconte le souvenir qu'il a du microscope quand il était petit et y figure une analyse du microscope. Il y a deux thèmes, le rêve et les objets qui «nous» envahissent. Pour elle, seuls les contenus sont pris en considération.

Ces trois exemples montrent comment les photocopies constituent des documents pour lesquels il existe une graduation selon les capacités de lectures et d'écritures des élèves:

- en lycée professionnel pour leur apprendre à lire des écrits complexes,

- en lycée technique pour les faire entrer dans des activités d'écritures sophistiquées,

- en brevet de techniciens du supérieur (BTS) pour lesquels le médium n'est plus perçu et dont la manipulation est implicitement considérée par l'enseignant.e comme incorporée.

De ce rapport à l'écrit des élèves découle une stratégie d'enseignement reflétée par la nature des documents produits: laisser une trace, les faire lire, les faire écrire, les intéresser pour les mettre en activité.

\section{Deux cas de non-usage des photocopies}

Lors de ce travail, nous n'avons rencontré que deux situations pour lesquelles les enseignant.e.s ne souhaitaient pas utiliser de photocopies. Le premier en sciences de la vie et de la Terre (SVT) pour un enseignement sur le développement durable et le second pour une classe dont le chahut rendait impossible un fonctionnement ordinaire.

Une enseignante de SVT stagiaire n'utilisait aucun document pour le thème de la classe de seconde Enjeux planétaires contemporains qui présente des enjeux écologiques. «Il a fallu que je revoie toute ma façon de faire... J'ai tout mis sur 
mon [diaporama]». Il s'agissait de sensibiliser les élèves à la consommation de papier, tout l'enseignement du thème, s'étalant sur plusieurs séances, s'est donc déroulé sans aucune photocopie. Elle a notamment été contrainte de faire plus souvent appel au manuel et de projeter au tableau des pages de livres contenant les documents à analyser. En l'absence de photocopies, les activités de classe ont changé: "on a fait un débat sur les biocarburants». Sur la partie portant sur la photosynthèse, elle a fait travailler les élèves selon la démarche expérimentale en alternant vidéoprojection et prise de notes des élèves. Si des photocopies avaient été mobilisées, les élèves auraient complété une fiche d'activité de travaux pratiques. Dans ce cas, le vidéoprojecteur est présenté comme un moyen de substitution.

Une classe de seconde générale d'une enseignante contractuelle de mathématiques chahutait beaucoup pendant ses cours. Lors de sa prise de poste, elle a d'abord estimé que les photocopies n'étaient pas importantes puis a rencontré beaucoup de difficultés. Pour l'aider ses collègues lui ont conseillé de préparer des supports visuels papier ou projetés et d'éviter les cours magistraux au tableau. Le professeur principal de cette classe est venu discuter avec elle en présence de l'enquêteur et, après qu'elle eut raconté le déroulement du chahut s'étant déroulé la veille, il lui a donné une série de conseils excluant l'usage de photocopies, sauf pour évaluer les élèves:

tu vidéoprojettes et tu dis «vous copiez !», [...] il faut arrêter de leur servir la soupe. À un moment donné «t'écris» et puis c'est tout, quoi! [...] Ils copient ce que tu as projeté et toi tu regardes et le premier qui moufte hop! [...] Ils ont juste à recopier ce qu'il y a au tableau il n'y a plus rien....

Ce qui était manifeste dans ces conseils c'était de limiter la présentation de documents en faveur d'une activité d'écriture contrainte.

\section{Discussion}

Les discours des enseignant.e.s que nous avons collectés se recoupent et mettent en évidence une cohérence dans ce qui est exprimé, le reflet de pratiques similaires. Cet élément nous conforte dans la validité des thématiques exposées ici.

Les documents photocopiés remis aux élèves sont des documents centraux dans l'activité enseignante. Les sources principales de conception des documents polycopiés sont les manuels scolaires et les résultats de recherche de Google. Les raisons déclarées d'emploi massif des photocopies sont d'abord l'absence de manuels scolaires pour le niveau enseigné et leurs inadaptations aux préférences des enseignant.e.s. Les photocopies constituent une adaptation des contenus enseignés aux capacités de lecture et d'écriture des élèves. De ce critère découle la stratégie d'enseignement et la nature des photocopies produites. Autrement dit, selon que les élèves ont besoin de s'exercer à lire ou à écrire les enseignant.e.s mettent en œuvre une stratégie d'enseignement qui repose sur la production d'un 
document polycopié spécifique. Lorsque les élèves sont à l'aise avec la lecture et l'écriture, le support n'est plus mentionné et la stratégie d'enseignement et de conception de la photocopie est focalisée sur les contenus uniquement.

Pour comprendre le statut et la fonction que jouent ces documents dans la classe, il faut considérer que le support de cours constitue la matérialisation de la position de l'enseignant.e en classe qui se trouve en quelque sorte entre les élèves et les savoirs à enseigner. Les contraintes induites par cette rencontre s'imposent aux enseignant.e.s qui doivent instrumenter leurs documents en classe de façon à ménager en priorité les élèves, mais en faisant aussi peu de concessions que possible sur la rigueur et l'exactitude des savoirs enseignés et, si possible, en prenant du plaisir à partager une connaissance qui leur semble utile. En somme, les photocopies sont un médium permettant d'établir un modus operandi entre les contraintes liées aux élèves, l'organisation scolaire quotidienne et les contenus à enseigner. Dès lors que l'on adopte cette perspective, ce que disent les enseignant.e.s sur les photocopies devient signifiant. Cela rejoint la perspective de Cèbe et Goigoux (2007): les technologies doivent être en accord avec les conceptions pédagogiques des enseignant.e.s sans négliger les caractéristiques des élèves et les connaissances à enseigner.

La comparaison de l'emploi des photocopieuses avec les technologies informatisées est intéressante d'abord en termes des moyens alloués (budget, personnels dédiés, locaux, fournitures...). Ensuite, la réalisation de patchworks, c'est-à-dire d'échantillons d'écrits, est facilitée par l'emploi des traitements de texte et des moteurs de recherche. Les documents photocopiés relèvent d'un processus technologique hybride (traitement de texte, reprographie, graphie manuscrite...) afin de s'intégrer aux technologies scolarisées (classeurs, cahiers, tableaux...). Les technologies utilisées pour les concevoir reposent sur des produits fournis par un petit nombre de très grosses multinationales. Les instruments d'éditions principaux sont Microsoft Word et les photocopieuses des lycées (souvent sous contrats avec Xerox). Word, Google et les photocopieuses sont des produits dont l'ergonomie est extrêmement travaillée et peuvent donner l'illusion d'une simplicité d'utilisation susceptible d'assujettir leurs utilisatrices et utilisateurs que l'on pourrait parfois qualifier de captives et captifs ergonomiques.

Labsence de manuels scolaires pour le niveau enseigné ou leur inadaptation aux préférences des enseignant.e.s ne leur posent pas de problèmes, ils et elles invoquent ou suggèrent l'exercice de leur liberté pédagogique au regard de leurs responsabilités et de leurs expertises. On peut d'ailleurs estimer que les photocopies remises aux élèves sont des micromanuels scolaires artisanaux, sur mesure, modifiables, réutilisable et éphémères. À noter que le format des manuels et des photocopies sont les mêmes: des documents modulaires comme décrits par Choppin (2005). 


\section{Perspectives}

Dans ses premiers cours dispensés au Collège de France, Bourdieu (Bourdieu, Champagne, Duval, Poupeau, \& Rivière, 2015), expose notamment l'importance des modes de catégorisation des sujets classants. Avec ce regard, on peut interpréter les désignations des enseignant.e.s vis-à-vis de leurs élèves comme un mode de classification. En considérant que les groupes sociaux classent les objets de leur environnement selon les mêmes critères pratiques que les humains, on pourrait prolonger cette étude en cherchant à examiner avec quels soins les documents sont produits au regard de l'opinion qu'ont les enseignant.e.s des élèves de leurs classes. Il s'agirait de regarder les archives des enseignant.e.s comme les objets de la maison kabyle de Bourdieu (1980) ou les constellations des cieux australiens de Mauss et Durkheim (Mauss, 1969). Dans cette logique, il serait important d'étudier en s'appuyant sur Goody (1979) comment l'emploi de la photocopieuse a fait évoluer le curriculum produit en focalisant sur la nature des documents photocopiés. On pourrait ainsi s'interroger sur la diversité ou l'homogénéité des structures, des présentations, mais surtout sur la nature des documents et les contenus les plus significatifs. Il s'agirait d'analyser les photocopies en fonction des contenus disciplinaires, des programmes, des conceptions que les enseignant.e.s ont de leurs objets d'enseignement et d'apprentissage.

L'importance des photocopies dans les lycées questionne fondamentalement le format des manuels. Il est possible de considérer que les éditeurs fournissent des matériaux bruts au format papier pour lesquels ils reçoivent une première rétribution avec l'achat du manuel puis une seconde par le biais des redevances sur les photocopies. On pourrait imaginer une situation analogue avec un environnement documentaire permettant d'effectuer des recherches dans des bases de données de diverses natures et de composer des documents remis aux élèves dans un instrument logiciel. Les technologies ayant évolué, on pourrait légitimement considérer que les manuels scolaires ne soient plus exclusivement des livres remis aux élèves et aux enseignant.e.s, mais aussi un environnement documentaire permettant aux enseignant.e.s de produire des documents selon leurs besoins. Alors le rôle des pouvoirs publics pourrait être d'organiser un écosystème documentaire garantissant l'interopérabilité et la circulation de fichiers financés par des budgets publics notamment en imposant l'emploi de normes ouvertes aux éditeurs intervenant dans l'école. Cela favoriserait le développement d'applications permettant d'aller chercher des contenus sur différentes sources et de produire des documents numériques ou imprimés.

Dans ce processus sophistiqué, on a remarqué qu'il n'est presque jamais fait mention des centres de documentation et d'information (CDI). On pourrait imaginer qu'ils jouent un rôle d'indexation et d'archivage des documents produits par les enseignant.e.s et permettent ainsi la production d'une forme de capital d'établissement. Même si les enseignant.e-s partagent les documents qu'ils et elles produisent, ils et elles le font de façon considérée, des incitations 
diverses seraient susceptibles de formaliser et d'institutionnaliser ces échanges. En particulier, pour les jeunes enseignant.e.s, l'institution serait en mesure de leur fournir des exemples de documents issus de contextes similaires aux leurs, ils et elles hériteraient alors de documents leur permettant de réaliser les adaptations et ajustements à leurs classes.

\section{Références}

Balcou-Debussche, M. (2007). Rapports des enseignant.e-s aux formes de savoirs et à l'écriture vus à travers l'usage des photocopies à l'école. Revue Française de Pédagogie, 161, 15-23.

Boelaert, J., \& Khaneboubi, M. (2016). Éléments d'analyse de données produites par le centre français d'exploitation du droit de copie (CFC) (Livrable au Projet ReVEA). Repéré à http://eda.recherche.parisdescartes.fr/wp-content/uploads/sites/6/2019/03/rapport_2.3_ revea_photocop_quali_2017_def.pdf

Bourdieu, P. (1980). Le sens pratique. Paris, France: Les Éditions de Minuit.

Bourdieu, P., Champagne, P., Duval, J., Poupeau, F., \& Rivière, M.-C. (2015). Sociologie générale: Volume 1, Cours au Collège de France. Paris, France: Seuil.

Bruillard, É. (2019). Understanding teacher activity with educational resources. Selection, creation, modification, use, discussion and sharing. In J. Rodrígez Rodrígez, T. M. Braga Garcia, \& É. Bruillard, (Éds.), IARTEM 1991-2016: 25 years developing textbook and educational media research (pp. 343-352). Santiago de Compostela, Espagne: IARTEM.

Cèbe, S., \& Goigoux, R. (2007). Concevoir un instrument didactique pour améliorer l'enseignement de la compréhension de textes. Repères. Recherches en didactique du français langue maternelle, 35(1), 185-208.

Choppin, A. (2005). L'édition scolaire française et ses contraintes: une perspective historique. In E. Bruillard (Éd.), Manuels scolaires, regards croisés (pp. 39-45). Caen, France: ScérénCRDP Basse-Normandie.

Cuban, L. (1986). Teachers and Machines: The Classroom Use of Technology Since 1920. New York, NY: Teachers College Press.

Cuban, L. (2001). Oversold and Underused: Computers in the Classroom. Cambridge, MA: Harvard University Press.

Edgerton, D., \& Jeanmougin, C. (2013). Quoi de neuf?: du rôle des techniques dans l'histoire globale. Paris, France: Seuil.

Edgerton, D., \& Pestre, D. (1998). De l'innovation aux usages. Dix thèses éclectiques sur l'histoire des techniques. Annales. Histoire, Sciences Sociales, 53(4), 815-837.

Geertz, C. (1998). La description dense. Enquête, 6, 73-105.

Glaser, B., \& Strauss, A. (2010). La déconverte de la théorie ancrée. Stratégies pour la recherche qualitative. Malakoff, France: Armand Colin.

Goody, J. (1979). La raison graphique. La domestication de la pensée sauvage. Paris, France: Les Éditions de Minuit.

Horsley, M. (2012). Investing in classroom teaching and learning resources: access and equity in providing classroom teaching and learning materials in Australian schools. Sydney, Australia: Australian Publishers Association.

Horsley, M., \& Walker, R. (2011). It's all online: but is it enough? A case study of the development of a digital education repository. In Representations of otherness - IARTEM, sept 2011 Kaunas Lithuania (pp. 114-118). Rockhampton, Australia: Central Queensland University.

Khaneboubi, M. (2009). Facteurs influençant les usages de l'informatique en classe par des enseignant.e.s de collèges du département des Landes. In L.-O. Pochon, E. Bruillard, \& G.-L. Baron (Éds.), Informatique et progiciels en éducation et en formation : Continuités et perspectives (pp. 154-167). Lyon, France: INRP - IRDP - ENS de Cachan. 
Mauss, M. (1969). Oeuvres, tome 2: Représentations collectives et diversité des civilisations. Paris, France: Éditions de Minuit.

Mayor, G., \& Chevalier, S. (2008). La main à la plume vaut la main à la charrue. Entretien avec Jack Goody, première partie. ethnographiques.org, 16. Repéré à: http://www.ethnographiques.org/2008/Goody-Chevalier-Mayor

Nonnon, E. (2000). Le tableau noir de l'enseignant, entre écrit et oral. Repères. Recherches en didactique du français langue maternelle, 22(1), 83-119.

Plane, S., \& Schneuwly, B. (2000). Regards sur les outils de l'enseignement du français: Un premier repérage. Repères. Recherches en didactique du français langue maternelle, 22(1), 3-17.

Poffé, C., Laschet, M., \& Hindrycks, M.-N. (2015). Les supports pour l'étude fournis à l'élève en sciences biologiques sont-ils créateurs d'inégalités? Étude exploratoire de productions de futurs enseignant.e.s en Fédération Wallonie-Bruxelles (Belgique). Spirale. Revue de Recherches en Éducation, 55, 29-44.

Thévenaz-Christen, T. (2014). La lecture enseignée au fil de l'école obligatoire: L'exemple genevois. Namur, Belgique: Presses universitaires de Namur.

Thouvenin, M. (1975). Enquête sur les activités reprographiques dans les établissements ou centres d'enseignement et de documentation. Paris, France: I.N.R.D.P. (Institut national de recherche et de documentation pédagogiques).

Mots clés: Photocopies, enseignant·e, lycée, TIC, qualitatif, France

\section{Pädagogische Fotokopien: ein Werkzeug für die Lehrpraxis zur Angleichung der Lehrinhalte an die Lese- und Schreibfähigkeiten der Schüler}

\section{Zusammenfassung}

Eine Analyse der Aussagen von 46 Lehrer/-innen aus Gymnasien und Fachschulen weist darauf hin, dass diese die Erstellung von Fotokopien als eine Möglichkeit der Anpassung der Lehrinhalte an die Lese- und Schreibfähigkeiten der Schülerinnen und Schüler betrachten . Die Lese- und Schreibfähigkeiten der Schüler/innen sind für die Unterrichtsmethoden der Lehrer/-innen und die Art der Fotokopien ausschlaggebend. In Abhängigkeit von der Einschätzung der Lehrer/ innen über die Lese- und Schreibfähigkeiten ihrer Schüler/-innen, entwerfen sie unterschiedliche Dokumente, die dieser Einschätzung entsprechen. Wenn die Schüler/-innen gute Leser sind, werden diese Aspekte vernachlässigt und die Gestaltung des Unterrichts sowie das Design der Arbeitsblätter konzentriert sich hauptsächlich auf die Inhalte.

Schlagworte: Fotokopie, Arbeitsblätter, Lehrer, Gymnasium, ICT, qualitativ, Frankreich 


\title{
Le dispense: uno strumento educativo al servizio delle pratiche didattiche per adattare i contenuti insegnati alle capacità di lettura e scrittura degli allievi
}

\section{Riassunto}

L'interpretazione dei discorsi di 46 insegnanti di scuole superiori generali, tecnologiche e professionali, ci porta a considerare la produzione di documenti fotocopiati come un adattamento dei contenuti insegnati alle capacità di lettura e di scrittura degli allievi. Le capacità di scrittura degli allievi guidano la strategia didattica degli insegnanti e la natura delle dispense. Nel caso ritengano che i loro allievi abbiano bisogno di esercitarsi nella lettura o nella scrittura, progettano un documento adatto. Quando gli studenti sono buoni lettori, il mezzo diventa invisibile e la strategia di insegnamento e di progettazione delle dispense si concentra solo sui contenuti.

Parole chiave: Dispense, insegnante, scuola superiore, TIC, qualitativo, Francia

\section{Handouts: a tool for teacher teaching practices to adjust students' scriptural skills and the content being taught.}

\begin{abstract}
Discourse analyses of 46 teachers from general, technological and professional high schools makes us consider the use of handouts as an adaptation of the content taught to the reading and writing skills of students. Students' scriptural skills influence teachers' teaching strategies and the nature of handouts. Depending on whether they consider that their students need to practice in reading or writing, they design different documents fitted to their needs. When students are good readers, the printed media becomes invisible and the teaching and design strategy for the handout is focused on content only.
\end{abstract}

Keywords: Photocopy, teacher, high school, ICT, qualitative, France 


\section{Auteur.e.s}

Mehdi Khaneboubi est maître de conférence à l'université Cergy Paris. Ses recherches, ancrées dans les sciences de l'éducation, portent principalement sur les usages des technologies de l'information et de la communication par les enseignant·e·s. Au début des années 2000, au laboratoire DAEST de l'université Victor Segalen Bordeaux 2, il réalise sa thèse traitant des usages des ordinateurs portables par les enseignant.e.s de collège du département des Landes. À la fin des années 2000, en contrat, post doctoral au laboratoire EDA de l'université Paris Descartes, il étudie le projet de dotation en ordinateur portable du département de l'Oise et participera à des travaux sur les questions de genre dans l'enseignement des STEM. De 2013 à 2017, en détachement à l'institut français d'éducation (IFÉ) au laboratoire STEF, il s'engage notamment au projet ReVEA portant sur les ressources pédagogiques dont est issue cette contribution.

Laboratoire Sciences Techniques Éducation Formation (STEF), École normale Supérieure de Cachan, Bâtiment Cournot, 61, avenue du Président Wilson, F-94235 Cachan cedex

E-Mail: mehdi.khaneboubi@ens-cachan.fr

Camille Roux-Goupille, Maîtresse de conférences en biologie à l'université Paris Est Creteil et biologiste de formation, Camille Roux-Goupille a tout d'abord effectué des recherches et enseigné en biologie végétale. Après une reconversion thématique en histoire des sciences \& Didactique, elle a rejoint le laboratoire STEF de l'ENS Cachan, ce qui lui a permis de participer aux recherches en cours, notamment dans le cadre du projet ANR-ReVEA, concernant le travail des enseignant.e.s avec les ressources, que ce soit les processus de recherche, de sélection, de transformation et de création. Ayant depuis rejoint le laboratoire LDAR, elle s'intéresse maintenant à l'étude aux places et rôles de l'histoire des sciences dans l'enseignement de la biologie.

Camille Roux-Goupille, Laboratoire de Didactique André Revuz (LDAR), Univ Paris Est Creteil, LDAR, F-94010, Creteil France

E-Mail: croux@u-pec.fr 


\section{Annexe: Canevas d'enquête}

Présentation de l'enquête

Présentation de l'enquêteur...

Le projet ReVEA s'intéresse aux pratiques enseignantes vis-à-vis des ressources pédagogiques dans l'enseignement secondaire (conception, recherche, sélection, modification, recomposition...). C'est un projet de recherche financé pendant 4 ans par l'ANR et par 4 laboratoires de SHS.

La photocopieuse est une technologie plus installée et plus fiable que les outils numériques. On suppose que les documents photocopiés sont spécifiquement pensés pour des activités pédagogiques, et qu'ils reflètent un travail de composition/création particulier.

La question des droits d'auteurs n'entre pas dans le cadre de l'enquête.

Il s'agit d'un travail anonyme.

\section{Élément de contexte de L'établissement}

Quels types de formations sont dispensés dans le lycée? Y a-t-il des BTS, des classes prépa [CPGE]...? Combien y a-t-il d'enseignant.e.s et d'élèves dans l'établissement?

Dans quelles filières les élèves poursuivent-ils après le lycée? Où se trouve le lycée (grande ville, banlieue, campagne...)? Est-il classé ZEP?

Où sont localisées les photocopieuses? Existe-t-il aussi un service de reprographie? Qui a le droit de faire des photocopies? Y a-t-il des quotas?

\section{Avec le gestionnaire (ou intendant)}

Lorsque vous avez rempli l'enquête du CFC, combien d'enseignant.e.s y ont répondu?

Combien de photocopies sont faites par semaine ou par an?

Quel budget est ce que cela représente? Est-ce beaucoup par rapport au budget du lycée? Est-ce que les photocopies posent des problèmes?

Comment sont organisés le choix, l'acquisition, la distribution, le remplacement... des manuels scolaires? Est-ce que les ordinateurs, le réseau informatique fonctionnent bien? Y a-t-il le WiFi?

\section{Avec le responsable de la reprographie}

Comment fait un.e enseignant.e qui veut faire des photocopies? Quelle est la procédure (si elle existe)? Comment ça se passe? 
Quel type de photocopies faites-vous le plus souvent (manuel, livre...)? Quels types de documents photocopiez-vous le plus souvent? Est-ce que vous photocopiez beaucoup de montages?

Quelles sont les disciplines qui photocopient le plus?

Quelles évolutions dans les photocopies avez-vous vues ces dernières années (ou par rapport à l'établissement où vous vous trouviez avant)?

\section{Avec des enseignant-e.s}

Vous êtes prof de quoi? Depuis combien de temps? Combien de temps dans ce lycée? Quelles classes (et spécialités) avez-vous cette année?

Est-ce que les photocopies sont importantes? Si la reprographie était fermée comment feriez-vous? Est-il possible de se passer de photocopies? Si non pourquoi? Si oui comment feriez-vous? À quelle fréquence faites-vous appel à la reprographie?

Qu'est-ce que vous êtes venu photocopier? Accepteriez-vous de m'en montrer un exemplaire? Comment fabriquez-vous les montages que vous photocopiez? Quelle est l'origine de ces éléments? Avec quels logiciels les avez-vous produits? Avez-vous déjà utilisé tout ou partie de ce document auparavant?

Quelles sont vos sources de préparation: sur internet, en papier, etc.?

Est-ce que vous produisez des documents à plusieurs, en groupe (disciplinaire ou non)? Comment ça se passe? Quelles sont les pratiques habituelles de production de ressources? 